\title{
Survivin-Induced Aurora-B Kinase Activation
}

\author{
A Mechanism by Which APC Mutations Contribute to \\ Increased Mitoses during Colon Cancer Development
}

Tao Zhang, ${ }^{* \dagger}$ Jeremy Z. Fields, ${ }^{\ddagger}$ Lynn Opdenaker, ${ }^{\star}$ Tomas Otevrel, ${ }^{\dagger}$ Emi Masuda, ${ }^{\dagger}$ Juan P. Palazzo, ${ }^{\S}$ Gerald A. Isenberg, ${ }^{\text {I" }}$

Scott D. Goldstein, "Marc Brand," and Bruce M. Boman ${ }^{\star \dagger * * \dagger \dagger}$

From the Center for Translational Cancer Research," University of Delaware, Newark, Delaware; the Divisions of Genetic \& Preventive Medicine ${ }^{\dagger}$ and Colorectal Surgery, " the Department of Pathology, and the Kimmel Cancer Center,** Thomas Jefferson University, Philadelphia, Pennsylvania; CA*TX Biotechnology, Inc., Gladwyne, Pennsylvania; the Division of Colorectal Surgery," Rush University, Chicago, Illinois; and the Helen F. Graham Cancer Center, "H Christiana Care Health System, Newark, Delaware

APC mutations initiate most colorectal cancers (CRCs), but cellular mechanisms linking this to CRC pathology are unclear. We reported that wild-type $A P C$ in the colon down-regulates the anti-apoptotic protein survivin, and $A P C$ mutation up-regulates it, explaining why most CRCs display survivin overexpression and apoptosis inhibition. However, it does not explain another hallmark of CRC pathologyincreased mitotic figures and cell proliferation. Because survivin activates aurora-B kinase (ABK) in vitro, catalyzing mitosis, we hypothesized that in normal colonic crypts, APC controls ABK activity, while in neoplastic $A P C$-mutant crypts, ABK activity is up-regulated, increasing mitosis. We quantitatively mapped intracryptal distributions of survivin, ABK, and markers of activated downstream signaling and mitosis (INCENP, phospho-histone-H3, phospho-centromere-protein-A). In normal crypts, gradients for these markers, ABK:survivin:INCENP complexes, and ABK activity were highest in the lower crypt (inverse to the APC gradient). In neoplastic crypts that harbor $A P C$ mutations, proliferating (Ki-67+) cells and cells expressing survivin, ABK, and phospho-histone-H3 were distributed farther up the crypt. Hence, as cells migrate up neoplastic crypts, transitions be- tween cell phenotypes (eg, from stem to proliferating) appear delayed. In CRC cell lines, increasing wild-type APC, inhibiting TCF-4, or decreasing survivin expression down-regulated ABK activity. Thus, APC mutation-induced up-regulation of the survivin/ABK cascade can explain delayed crypt cell maturation, expansion of proliferative cell populations (including mitotic figures), and promotion of colon tumorigenesis. (Am J Pathol 2010, 177:2816-2826; DOI: 10.2353/ajpath.2010.100047)

Although several lines of evidence indicate that a mutation at the APC locus initiates most cases of colorectal cancer (CRC), much less is known about the subsequent molecular and cellular mechanisms that link this mutation to the pathophysiology of colon tumorigenesis. Investigating this link by studying the anti-apoptotic protein survivin, we found that wild-type $A P C$ down-regulates survivin expression ${ }^{1}$ and mutation of $A P C$ up-regulates it in mouse ${ }^{2}$ and man. ${ }^{3}$ While this might explain why most colon tumor cells show increased survivin expression and inhibition of apoptosis, it does not explain the increased mitotic figures and cell proliferation that are also pathological hallmarks of tumors. Since experiments using cultured cells have shown that survivin activates $A B K, 4,5$ which catalyzes mitosis, and since several lines of evidence suggest that $A B K$ is involved in tumorigenesis, ${ }^{6-10}$ we hypothesized that: (i) in normal human colonic crypts wild-type APC down-regulates ABK

Supported in part by NIH grant 5R21DK62146 to B.B. It was also funded in part by a generous gift from Gregg and Stacey Bacchieri.

Accepted for publication August 3, 2010

A guest editor acted as editor-in-chief for this manuscript. No person at Thomas Jefferson University was involved in the peer review process or final disposition for this article.

Address reprint requests to Bruce M. Boman. M.D., Ph.D., MSPH Director, Cancer Genetics and Stem Cell Biology, Center for Translational Cancer Research, Helen F. Graham Cancer Center, Christiana Care Health System, Department of Biological Sciences, University of Delaware, 4701 Ogletown Stanton Road, Suite 1205B, Newark, DE 19713. E-mail: BrBoman@christianacare.org. 
activity and (ii) in neoplastic crypts, where APC is mutant, ABK activity becomes up-regulated and is associated with increased mitosis and proliferation.

To test this hypothesis, we designed a multipronged approach. This approach takes advantage of the availability of colonic tissues containing $A P C$ mutations during the various phases of CRC development. Thus, we investigated four types of tissues: (a) normal colonic crypts, (b) normal-appearing FAP crypts, (c) adenomas, and (d) colon carcinomas.

Therefore, in our first approach, we used quantitative immunohistochemical mapping to establish whether activation of the ABK mechanism downstream to survivin's signaling pathway quantitatively correlates with the distribution of proliferative cells, particularly mitotic cells, in normal colonic crypts. We previously reported that, in the normal colonic crypt, survivin is expressed in a gradient fashionbeing highest in the lower crypt-which is where proliferating cells, including mitotic cells, are located. ${ }^{1}$ This is consistent with the fact that the expression of survivin is highest during M-phase of the cell cycle and has a role in cell division. Similarly, it has been shown ${ }^{11}$ that there is an inverse gradient of $A P C$ - from negligible at the crypt bottom to highest at the crypt top-which parallel changes in the maturation of crypt cells as they migrate upwards along the crypt axis. Hence, here we quantitatively mapped expression of markers for survivin, ABK, ABK's enzymatic activity, proliferative cells (Ki-67) and mitosis (phospho-histone $\mathrm{H} 3$ ) at different levels of the colonic crypt, and compared them to APC's intracryptal expression pattern.

Since the binding of survivin and INCENP to ABK has been shown to activate ABK's enzymatic activity in vitro, which catalyzes mitosis, our second approach was to determine whether this mechanism occurs in vivo and if $\mathrm{ABK}$ activity parallels the intracryptal distribution of proliferative and mitotic cells in normal colonic epithelium. Consequently, we used immunoprecipitation analysis and ABK enzyme assays to evaluate if: (1) ABK binds to survivin and its other binding partner, INCENP, and (2) the consequence of binding is ABK activation and phosphorylation of its substrates, histone $\mathrm{H} 3$ and centromere protein A (CENP-A). CENP-A is an essential histone $\mathrm{H} 3$-like kinetochore protein incorporated at active centromeres.

Once we established that ABK-related mechanisms downstream of survivin are regulated by APC in normal colon, we then investigated whether survivin-induced Aurora- $\mathrm{B}$ kinase activation is a mechanism by which $A P C$ mutations might contribute to colon cancer development. We found that mutation of $A P C$ leads to up-regulation of survivin in neoplastic intestinal tissues in mouse ${ }^{2}$ and man. ${ }^{3}$ We also reported $^{1}$ and others confirmed ${ }^{12}$ that expression of the anti-apoptotic protein survivin is down-regulated by $\beta$-catenin/TCF-4 signaling, the activity of which is negatively controlled by APC. That survivin is a TCF- 4 target gene has also been confirmed by chromosome immunoprecipitation analysis $^{13}$ showing that TCF-4 binds to survivin's promoter region. Consequently, for our third approach, we investigated whether increasing wildtype APC, inhibiting TCF-4, or decreasing survivin expression in colon cancer cells (ones with mutant $A P C$ and survivin overexpression) reduces $A B K$ activity and cell proliferation.

Our fourth approach was to immunohistochemically map crypt cell populations and determine how they change during colon tumorigenesis. Our previous studies on mechanisms involved in the stepwise development of CRC indicate that dysregulation of survivin expression is a mechanism that contributes to the expansion of proliferative cell populations_-including stem cells (SCs) and proliferating cells. ${ }^{1-3}$ This and several modeling studies we did $^{14-16}$ led to the suggestion that pathological changes during coIon tumorigenesis can be explained by changes in SCs that alter the dynamics of the SC population and all other crypt cell populations. For example, in normal colonic crypts, homeostasis is maintained by SCs that reside at the crypt bottom and that generate proliferating cells that differentiate while migrating up the crypt axis. Our study ${ }^{1}$ suggested that wild type $A P C$, by progressively decreasing survivin and increasing apoptosis from crypt bottom to top, limits the size of the SC population and of other proliferative cell populations in the lower crypt. In the current study, we investigated the role of survivin in increased mitosis and proliferation in the development of CRC.

In colonic crypts of FAP patients, individuals who have a CRC-initiating, germline APC mutation, the population of proliferating cells is shifted toward the crypt top (as indicated by the labeling index), ${ }^{17-20}$ which indicates that maturation of cells is delayed as cells migrate up the premalignant crypt axis. Our study of FAP crypts ${ }^{2}$ and $\mathrm{Apc}^{\mathrm{Min} /+}$ mouse crypts $^{3}$ indicated that mutation of $A P C$ allows survivin to be overexpressed and proliferative cell populations to expand, thereby contributing to initiation of tumorigenesis. In this view, dysregulation of mechanisms that control crypt proliferative fraction ${ }^{16}$ explains how APC mutations (i) induce SC overpopulation at the crypt bottom, (ii) shift the proliferating cell population upwards, and (iii) initiate and promote colon tumorigenesis.

Consequently, in our fourth approach, we designed experiments 1) using quantitative immunohistochemistry to map crypt cell populations that express survivin signaling components and markers for cell proliferation, and 2) to determine whether and how these cell populations change during CRC initiation and progression.

\section{Materials and Methods}

\section{Tissue Procurement/Preparation of Whole Crypts}

Samples of normal human colon tissue were obtained from the distal margin of resection from individuals undergoing colon surgery, including, but not limited to, colon tumor resections. We investigated four types of tissues: (a) normal colonic crypts, (b) normal-appearing FAP crypts, (c) adenomas, and (d) colon carcinomas. Crypts were isolated from normal colon using a method we previously described. ${ }^{21}$ Crypt subsections (top, middle, bottom) were obtained by sequentially exposing coIonic mucosa to chelation solutions having increasing EDTA concentrations as described previously. ${ }^{1}$ Crypt 
subsection quality was checked by inverted phase microscopy.

\section{Inhibiting Survivin Signaling \\ Induction of Wild-Type APC}

This was done as we previously described ${ }^{1}$ using the colon carcinoma cell line HT29 containing a zinc-inducible APC gene (HT29-APC) and a control cell line containing an analogous inducible lacZ gene (HT29-Gal). Expression of full-length APC was induced with 120 $\mu \mathrm{mol} / \mathrm{L} \mathrm{ZnCl}_{2}$ for the times indicated. Endogenous phospho-H3 and phospho-CENP-A levels and the ability of immunoprecipitated-ABK to phosphorylate exogenous H3 were measured (described below).

\section{Transfection of Dominant Negative (dn) TCF-4}

The dnTCF-4 construct was made as previously described. ${ }^{1}$ The protein encoded by the plasmid is a competitive inhibitor of Tcf4 signaling -it does not interact with the endogenous Tcf-4 transcription factor; rather, it causes transcriptional suppression of Tcf4's downstream targets. The plasmid's expression cassette was designed to produce a transgenic Tcf-4 protein that is identical to the endogenous Tcf-4 protein except that the DNA binding region is absent. Since the expressed transgenic protein competes with the endogenous Tcf-4 protein for binding to $\beta$-catenin, the complex formed between $\beta$-catenin and the truncated form of Tcf-4 cannot bind to DNA. Thus, endogenous Tcf-4 itself is not affected by the dnTcf-4 protein expressed from the transfected plasmid.

The dnTCF-4 construct was transfected transiently into the HT29 cell line using a lipofection technique as previously described. ${ }^{1}$ To generate stable transfections, $2 \mu \mathrm{g}$ of linearized DNA plasmid constructs were introduced into cells and 48 hours after transfection cells were trypsinized and plated into medium containing G418 (Gibco BRL, Grand Island, NY). Stable transfectants were harvested 10 days after the beginning of transfection. Endogenous phospho-H3 and phosphoCENP-A levels and the ability of immunoprecipitatedABK to phosphorylate exogenous $\mathrm{H} 3$ were measured (described below). The control used in the study was the empty plasmid.

\section{Inactivation of Survivin by Small Interfering RNA}

HT29 cells in exponential growth were seeded onto 24-well plates at $1 \times 10^{5}$ cells per well and grown overnight in Dulbecco's modified Eagle's medium/10\% fetal bovine serum/0.1 mmol/L Non-Essential Amino Acid medium (Gibco-BRL) and maintained in $5 \% \mathrm{CO}_{2}$ at $37^{\circ} \mathrm{C}$. The cells were transfected at $50 \%$ confluency. RNA interference transfection was performed according to the protocol provided by Invitrogen. Briefly, 50 pmol of siRNA were incubated with $3 \mu$ l Lipofectamine 2000 in OptiMEM Reduced Serum Media (Invitrogen) to form com- plexes. The cell medium was replaced with Dulbecco's modified Eagle's medium without fetal bovine serum 0.1 $\mathrm{mmol} / \mathrm{L}$ Non-Essential Amino Acid and antibiotics. The complexes were administered to the cells and incubated for 5 hours in $5 \% \mathrm{CO}_{2}$ at $37^{\circ} \mathrm{C}$. After 5 hours, the medium was adjusted to the normal serum concentration, and cells were maintained under regular growth conditions until harvesting. Cells were assayed for TCF-4 inhibition by immunoblotting. Cyclophilin B-targeting siRNA (Dharmacon) was used as a positive control. siTOX Transfection Reagent (Dharmacon) was used as a control for transfection efficiency. Functional, nontargeting siRNA (Dharmacon) was used as a control for nonspecific effects associated with small interfering (si)RNA delivery.

\section{Reverse Transcription-PCR Amplification}

RNA extraction, cDNA synthesis and reverse transcriptionPCR were done as we previously described. ${ }^{1}$ The primers used to detect fragments of the ABK gene were designed from published human sequences (BC000442). The sequences were: AAGGAGCTGCAGAAGAGCTG and GGCGATAGGTCTCGTTGTGT, giving an amplified product of 363 bp. Negative controls included replacing RNA or cDNA with distilled water. To confirm the integrity of cDNA, fragments of the housekeeping gene $\beta$-actin were amplified concurrently. The sequence of the cDNA was compared to that of the gene bank and they were found to be identical.

\section{Immunohistochemistry/Immunofluorescence}

Immunohistochemistry using $5-\mu \mathrm{m}$ tissue sections was done as we previously reported. ${ }^{1,3}$ Primary antibodies were: anti-survivin (1:400 dilution; Novus Biologicals, Littleton CO); anti-ABK polyclonal antibody (1:100; Novus); antiKi-67 (1:100; Immunotech, Westbrook, ME); anti-phospho-H3 antibody (1:100; Upstate, Inc; Lake Placid, NY). For immunofluorescence, slides were incubated with primary antibody and stained with a fluorescently conjugated lgG (1 hour; $\left.24^{\circ} \mathrm{C}\right)$. Double staining was done for survivin and ALDH1 (1:50; BD Pharmingen), a marker for colonic SCs. ${ }^{22}$ Slides were washed, mounted with Prolong Gold anti-fade reagent (Invitrogen), and coverslipped. Slides were viewed with a Zeiss LSM 510 Meta confocal microscope (Carl Zeiss, NY).

\section{Staining Indices}

Staining indices were determined as we previously reported. ${ }^{22}$ Graphical display of indices and curve fitting (using sixth order polynomial analysis) were done using Excel (v. 2002, Microsoft, Redmond WA). We did not plot any indices for carcinomas because they do not contain recognizable crypt structures.

\section{Immunocytochemistry of Cultured Cells}

The slides containing cultured cells were prepared with a culture chamber slide (Nalge Nunc International, Naper- 
ville, IL). After growing a layer of cells on the slide, slides were washed thoroughly with five changes of PBS for 2 minutes each. Cold acetone was added to the cells for 10 minutes at $-20^{\circ} \mathrm{C}$ to fix them. Slides were then incubated in a solution of 0.25 Triton $X-100,5 \%$ dimethyl sulfoxide in PBS for 10 minutes to permeablize the cell membrane. The remaining methods were similar to those described above for immunohistochemistry of colon tissue.

\section{ABK Immunoprecipitation}

Crypt subsections or cells were lysed by lysis buffer (150 $\mathrm{mmol} / \mathrm{L} \mathrm{NaCl}, 50 \mathrm{mmol} / \mathrm{L}$ Tris pH7.5, 1\% NP40, 0.1\% sodium deoxycholate, $0.1 \%$ SDS, $1 \mathrm{mmol} / \mathrm{L}$ EDTA, 1 $\mathrm{mmol} / \mathrm{L}$ EGTA, $2 \mathrm{mmol} / \mathrm{L}$ phenylmethylsulfonyl fluoride, protease inhibitor mixture, and phosphatase inhibitor mixture (Sigma, St. Louis, MO). After centrifugation at $12,000 \times g$ for 10 minutes at $4^{\circ} \mathrm{C}, 1000 \mu \mathrm{g}$ of protein from the supernatant was pre-cleared at $4^{\circ} \mathrm{C}$ for 60 minutes with $10 \mu \mathrm{g}$ normal mouse IgG and $50 \mu \mathrm{l}$ of $50 \%$ proteinA-Sepharose CL-4B slurry (Sigma). The pre-cleared lysates were incubated with $2 \mu \mathrm{g}$ of AIM-1 (murine $A B K$ ) antibody (BD Bioscience, San Jose, CA) at $4^{\circ} \mathrm{C}$ for 2 hours with rocking. The immuno-complexes were precipitated with 50\% protein-A-Sepharose CL-4B (50 $\mu \mathrm{l}$ ) for 60 minutes at $4^{\circ} \mathrm{C}$ with continuous mixing and washed four times with wash buffer $(150 \mathrm{mmol} / \mathrm{L} \mathrm{NaCl}, 50 \mathrm{mmol} / \mathrm{L}$ Tris $\mathrm{pH} 7.5,0.1 \%$ NP40, $0.01 \%$ sodium deoxycholate, $0.1 \%$ SDS, $1 \mathrm{mmol} / \mathrm{L}$ EDTA, $1 \mathrm{mmol} / \mathrm{L}$ EGTA, $2 \mathrm{mmol} / \mathrm{L}$ phenylmethylsulfonyl fluoride).

\section{Aurora-B Kinase Assays}

The kinase activity of ABK was analyzed following the protocol of Upstate Inc. (Lake Placid, NY). Briefly, Sepharose beads from the aforementioned immunoprecipitation were suspended in $10 \mu \mathrm{l}$ Tris Assay Dilution Buffer (Upstate). One $\mu \mathrm{g}$ Histone H3 (Upstate) and $5 \mu \mathrm{l}$ of magnesium/ATP cocktail (Upstate) were added and incubated for 10 minutes at $30^{\circ} \mathrm{C}$. An equal volume of $2 \times$ Laemmli sample buffer (120 mmol/L Tris, pH6.8, 4\% SDS, 20\% glycerol, 10\% ßmercaptoethanol, $0.005 \%$ bromophenol blue, $0.5 \mathrm{mmol} / \mathrm{L}$ phenylmethylsulfonyl fluoride) was added and the mixture was then boiled for 5 minutes. The sample was loaded and run on a $12 \%$ SDS-polyacrylamide gel and then transferred to a polyvinylidene difluoride membrane. Polyvinylidene difluoride membranes were then blocked in freshly prepared TBS containing 3\% bovine serum albumin and $0.1 \%$ Tween 20 for 1 hour at room temperature. The membranes were incubated with $1 \mu \mathrm{g} / \mathrm{ml}$ of anti-phospho-histone-H3 antibody (Upstate) for 2 hours at room temperature. The membrane was then washed three times with water, and incubated with phosphatase-conjugated goat anti-rabbit antibody (1:5000) for 60 minutes, and developed with a substrate reagent kit (Bio-Rad, Hercules, CA). Negative controls contained no immunoprecipitation beads. Active ABK (Upstate) was used as a positive control.

\section{Western Blot Analysis}

This was done as we previously described, ${ }^{1}$ using antibodies against ABK (BD Bioscience), survivin (Novus), phospho-CENP-A, phospho-H3 (Upstate), or INCENP (from Dr. WC Earnshaw). As a control, B-actin protein was blotted concurrently. All experiments were repeated at least three times.

\section{Experiments Demonstrating that ABK Activity Mediates in Vitro Cell Proliferation in Cells with Mutant APC}

SW480 or HT29 cells were plated (25,000 cells/well in 12 well plates) and allowed to attach for 24 hours before 24 hours of serum starvation. Cells were then treated with various doses of ZM447439 (Tocris Bioscience, Ellisville, $\mathrm{MO}$ ) in full medium for 24 hours. Following determination of the IC-50 values, the assay was repeated with the indicated doses and cell number was determined every 12 hours using a trypan blue exclusion assay.

\section{Results}

Changes in the Intracryptal Distribution of Cells Expressing $A B K$ and its Binding Proteins Parallel Changes in Proliferation during Colon

\section{Tumorigenesis}

Here we did quantitative immunohistochemical mapping of the expression of $\mathrm{ABK}$ and its binding proteins in normal and malignant colonic crypts.

\section{Expression of ABK in Normal Colonic Crypts}

Immunohistochemistry was done using normal colonic epithelium to evaluate the expression of ABK. The results (Figure 1) show that the greatest proportion of cells showing ABK positivity was located in the lower crypt. A few cells at mid-crypt also showed staining, but none at or near the top did. Experiments on normal tissues showed that ABK staining was nuclear and positively stained cells were largely restricted to the bottom-third of crypts, where proliferating, $\mathrm{Ki}-67+$, cells are located. Although largely restricted to the bottom third of crypts, ABK staining marked fewer cells at the bottommost crypt levels (1 to 10), where colonic SCs reside. A similar pattern was seen for survivin. Quantitative mapping profiles (indices) derived from the immunohistochemistry data are shown in Figure 2 and confirm the qualitative results from immunohistochemistry staining seen in Figure 1. Double staining revealed that survivin and the colonic SC marker ALDH1 did not co-stain the same cells (Figure $3 A$ ). These patterns for $A B K$ and survivin were the inverse of the APC gradient (Figure 3, B and C).

To confirm that $A B K$ is preferentially expressed in the lower crypt, we used Western blot analysis of ABK levels in top, middle, and bottom subsections of normal human colonic crypts. Western blots (Figure 4A) also showed 

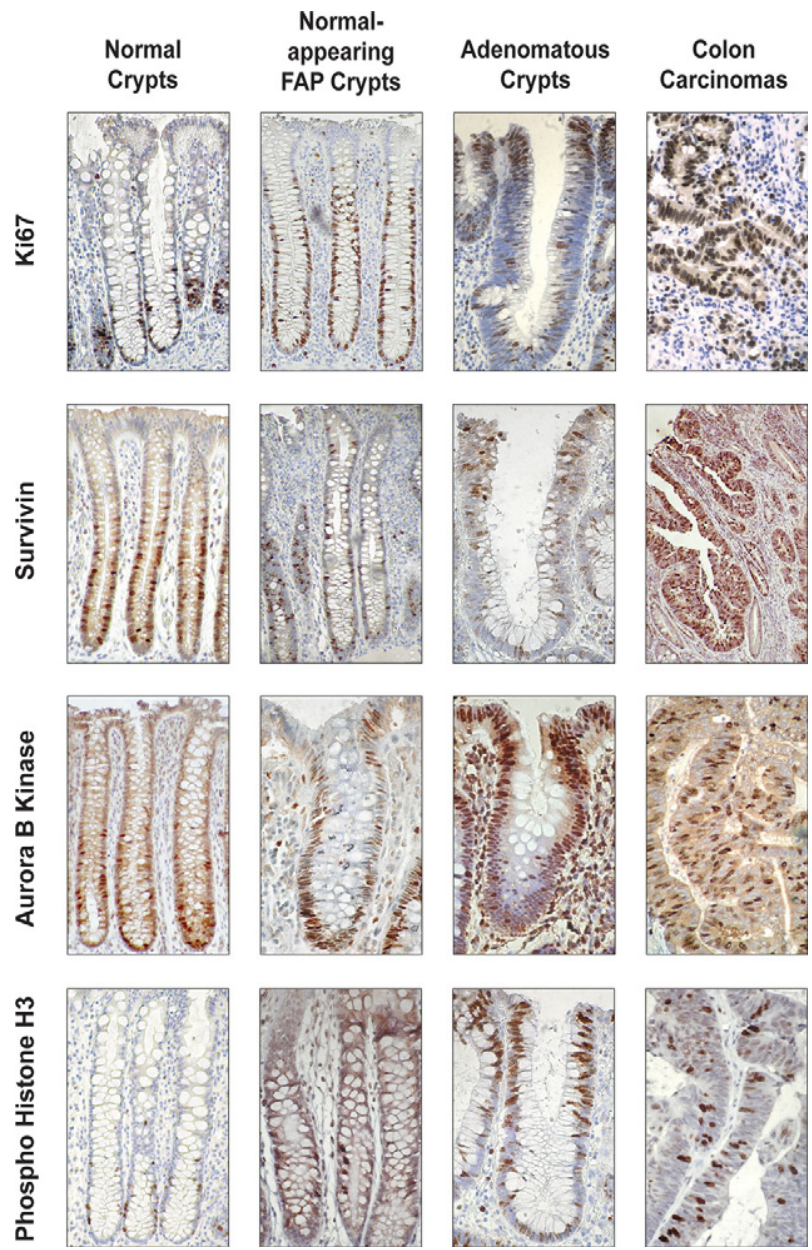

Figure 1. Immunohistochemical staining for Ki-67, survivin, ABK, and phospho-H3. Immunohistochemical analysis of the expression of Ki-67, survivin, $\mathrm{ABK}$, and phospho-H3 in normal colonic mucosa (left column), normalappearing colonic mucosa from FAP patients (center left column), adenomas (center right column), and colon carcinomas (right column).

that $A B K$ expression was highest in the crypt bottom and decreased toward the crypt top.

Expression of Survivin, ABK, Phospho-H3, and Ki-67 in Neoplastic Colonic Tissues

In normal crypts (Figure 1) the population of cells staining positively for $A B K$ was largely restricted to the bottom-third of crypts where proliferating (Ki-67+) and mitotic (phospho-H3+) cells are found; in normal-appearing tissue from FAP crypts, the population of $\mathrm{ABK}+$ cells extended upward into the crypt middle. In adenomas, the shift was even more pronounced$\mathrm{ABK}+$ cells were mostly found at the top of the crypt or on the luminal surface of the adenomatous epithelium. Similar changes in staining patterns were seen for survivin, phospho-H3, and Ki-67 (Figure 1). In contrast, most cells staining for the stem cell marker ALDH1 reside at or near the base of the normal colonic crypt. ${ }^{22}$ In FAP and adenomatous crypts, both the number of $\mathrm{ALDH}+$ cells and the extent of their distribution upward from the crypt bottom were increased. This expansion
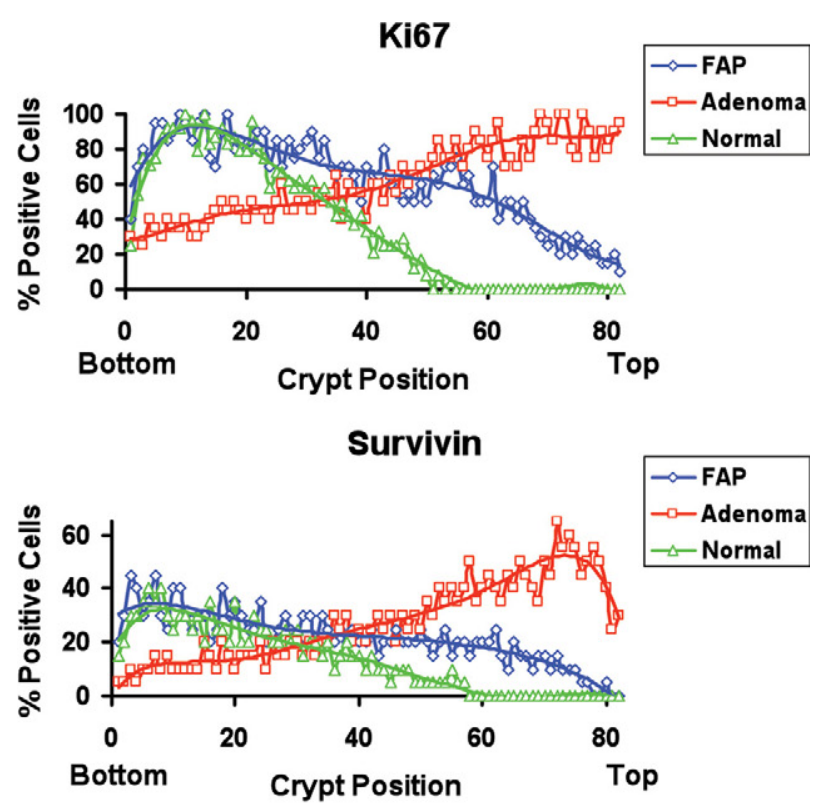

Aurora B Kinase

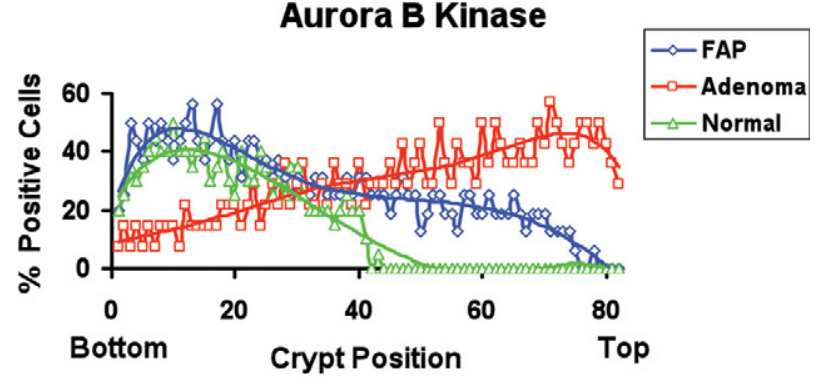

Phospho-Histone H3
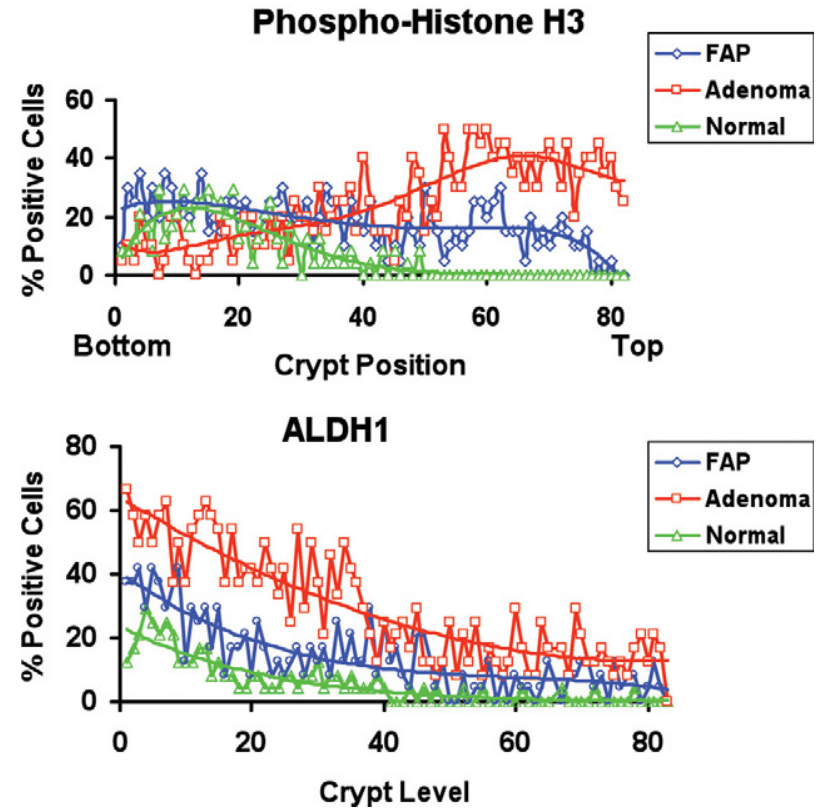

Figure 2. Staining indices for Ki-67, survivin, ABK, phospho-histone $\mathrm{H} 3$, and ALDH1. In normal crypts, survivin and its downstream signaling effectors (ABK and phospho-H3) showed similar profiles and localization to the lower crypt. Ki-67 staining indicated a similar profile for proliferating cells. During the progression from normal colon to adenoma, these positively staining cell populations expanded and shifted upward along the crypt axis. While the ALDH+ population also expanded, most of these cells remained lower in the crypt relative to cells staining for survivin and its downstream signaling effectors. 
A

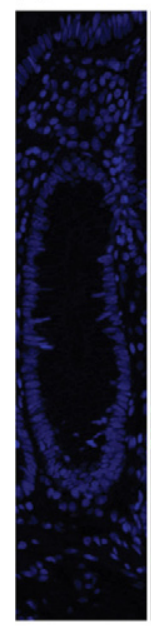

DAPI
Double Staining

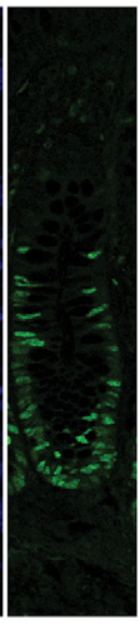

Survivin

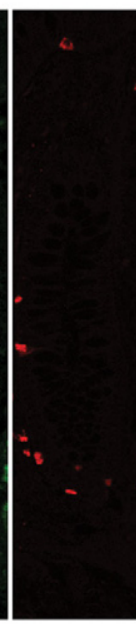

ALDH1

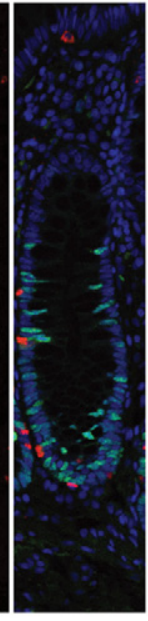

Merged
B Immunohistochemistry for APC

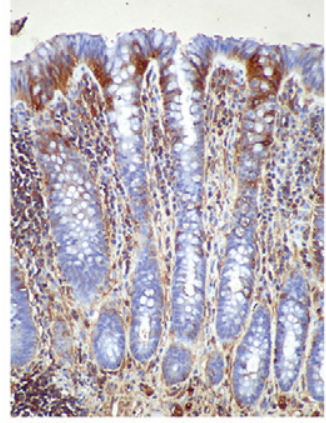

C Immunoprecipitation Western Blots

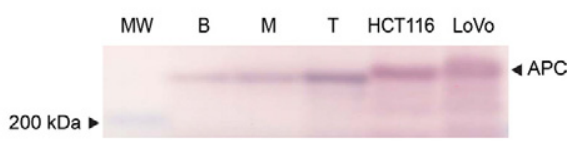

Figure 3. Double staining for survivin and the stem cell marker ALDH1, and analysis of APC expression by immunohistochemistry and immunoprecipitation/western blotting in norma colonic crypts. A: Double staining of normal colonic crypts (survivin-green; ALDH1-red) showed ALDH1 staining in the bottom of the crypts and survivin staining in the lower crypt, but these markers did not overlap. B: APC immunoreactivity in normal colonic crypts occurred at the crypt top and lumenal surface. $\mathbf{C}$ Immunoprecipitation of APC followed by western blot analysis for APC showed that its highest levels were in the normal crypt bottom (B) compared to middle crypt (M) and crypt top (T) Lane 1 was loaded with molecular weight (MW) markers. Positive controls involved analysis of HCT 116 cells and LoVo cells that express wildtype APC. Densitometry showed that, compared to the crypt top, APC expression was decreased by $50 \%$ in the middle crypt region and by $81 \%$ in the crypt bottom. of the ALDH+ cell population paralleled the upward shift of the other markers; however, the ALDH+ cell population remained considerably closer to the crypt base.

Indices (Figure 2) showed that during the stepwise progression to colon cancer (from normal colonic epithelium transitions to normal-appearing FAP-epithelium, and then to adenomatous epithelium), ABK expression extended further up the crypt. Indices for phospho-H3 and survivin showed similar changes. All changes in survivin signaling components paralleled changes in $\mathrm{Ki}-67$ staining indices. In carcinomas, where crypt structures can no longer be seen, cells staining positively for ABK, survivin, phospho$\mathrm{H} 3$, and Ki-67 were diffusely distributed throughout the tumor (Figure 1).

\section{ABK's Enzymatic Activity Indicates Downstream Survivin Signaling Is Activated in Normal and Neoplastic Colonic Tissues}

Here we used three independent approaches (see 1-3 below) to establish survivin-induced ABK activity in normal colonic crypt subsections and studied (see 4, below) the effects of inhibiting ABK activity on the proliferation of malignant colonic cells.

\section{Presence of ABK/Survivin/INCENP Complexes in Normal Colonic Crypt Subsections}

Previous in vitro studies on cultured cell lines ${ }^{4,23}$ demonstrated that ABK, survivin, and INCENP form a protein complex, which is associated with activated $A B K$ and mitosis. Our analysis of primary normal human colonic epithelium (Figure 4B) shows that survivin, INCENP, and $A B K$ exist in such a complex in vivo. This was done using co-immunoprecipitation with $\mathrm{ABK}$ antibody followed by western blot analysis with antibodies against $A B K$, survivin or INCENP. The gradient for the complex, as for survivin, INCENP, or ABK, was highest at the crypt bottom and lowest at the top.

\section{Phosphorylated Histone-H3 and CENP-A in Normal Colonic Crypt Subsections}

Since histone-H3 and CENP-A are substrates of $\mathrm{ABK},{ }^{8,24}$ we determined levels of phosphorylated forms of these proteins, in crypt subsections, as a measure of ABK activation. Western blot analysis showed (Figure 4A, rows 2 and 3) that endogenous phospho-histone-H3 and phospho-CENP-A were highest at the crypt bottom and lowest at the top.

\section{ABK Enzymatic Activity in Isolated Normal Colonic Crypt Subsections}

Phosphorylation of serine 10 of histone-H3 is required during mitosis for chromosome condensation and segregation and serves as a marker for ABK activation. 9,25,26 We immunoprecipitated, using anti-ABK antibody, ABK protein from crypt subsections and assayed for its enzymatic activity to phosphorylate exogenous histone-H3. Figure 4C shows that within the three crypt subsections, the lower crypt contained the highest ABK activity while the upper crypt had the least.

\section{Effects of ABK Activity on CRC Cell Proliferation}

Figure 5 shows the effects of a known ABK inhibitor on in vitro cell proliferation in cells with mutant $A P C$ and survivin overexpression (SW480 and HT29 cells). ZM447439, a known ABK inhibitor, decreased the in vitro growth of both colon cancer cell lines. While the inhibition of cell proliferation was more evident in HT29 cells, there were significant decreases in cell proliferation and survival in each cell line after treatment. 
A

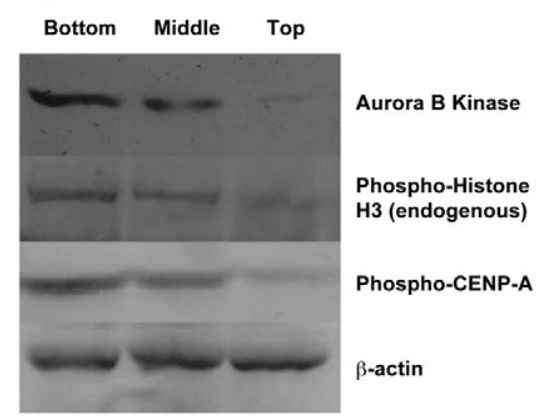

B

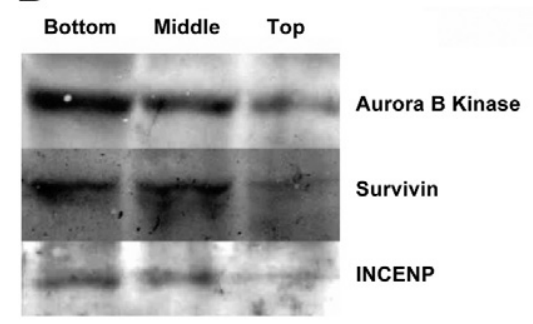

\section{C}

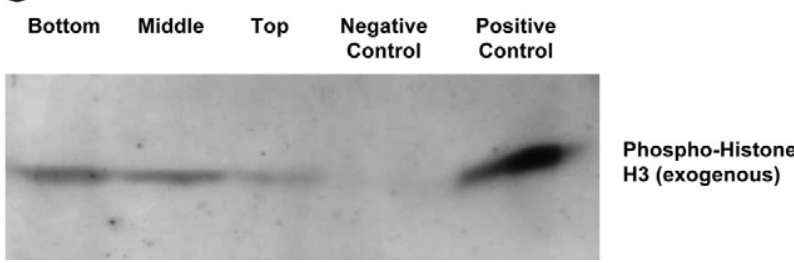

Figure 4. Expression of ABK, phospho H3, and phospho-CENP-A; presence of ABK:Survivin:INCENP protein complexes; and ABK activity in subsections (bottom, middle, top) of normal, human colonic crypts. A: Western blot analysis showed that ABK expression was highest in the crypt bottom and lowest in the upper crypt. Row 4 shows a $\beta$-actin control confirming that crypt subsection lysates contain equal amounts of protein. Gradients for ABK, phospho H3, and phospho-CENP-A were bottom $>$ middle $>$ top. Densitometry showed that, compared to the crypt bottom, the expression of ABK, phospho $\mathrm{H} 3$, and phosphor-CENP-A decreased by $64 \%, 61 \%$, and $51 \%$, respectively, in the middle crypt region; the expression of $\mathrm{ABK}$, phospho $\mathrm{H} 3$, and phosphor-CENP-A decreased by $87 \%, 96 \%$, and $94 \%$, respectively, in the crypt top. B: Immunoprecipitation analysis was also performed to detect ABK:Survivin:INCENP protein complexes in subsections (bottom, middle, top) of normal human colonic crypts. Immunoprecipitation with ABK antibody was followed by Western blot analysis of the same immunoprecipitation sample with anti-ABK, anti-survivin, and anti-INCENP antibodies. ABK was associated with survivin and INCENP in a protein complex. C: Immunoprecipitated ABK was also enzymatically active, and ABK activity within the three subsections of the crypt was greatest in the lower crypt and lowest in the upper crypt. Negative controls excluded immunoprecipitation beads (data not shown) and included a nonspecific control to monitor nonspecific binding of ABK to the beads. This latter control involved immunoprecipitating cell lysates with beads coupled with normal mouse IgG, which did not reveal any nonspecific binding of ABK as analyzed by polyacrylamide gel electrophoresis (data not shown) or any ABK enzyme activity (C). Positive controls included the addition of exogenous active ABK (C). Densitometry showed that, compared to the crypt bottom, ABK activity was decreased by $41 \%$ in the middle crypt region and by $79 \%$ in the crypt top.

\section{Inhibiting APC/ $\beta$-Catenin/TCF-4/Survivin Signaling Upstream Attenuates Survivin-Induced ABK Activation Downstream}

Here, we used three independent approaches_-increasing wildtype-APC, inhibiting TCF-4, or decreasing survivin expression in colon cancer cells (ones with mutant $A P C$ and survivin overexpression):

\section{Induction of Wild-Type APC}

We first established whether induction of wild type APC expression in HT-29 cells (that harbor mutant APC and overexpress survivin) down-regulates survivin expression. In the immunohistochemical analysis of cultured HT29-APC cells (containing a zinc-inducible wild-type APC gene), strongly positive survivin immunostaining that had been located in the cytoplasm before zinc induction of $A P C$ expression became weak after induction (Figure 6A). Although some residual survivin staining was still detectable 12 hours after induction of $A P C$, it was significantly reduced compared to control HT-29-Gal cells, which showed no difference in survivin immunoreactivity even 12 hours after exposure to zinc (data not shown). After zinc induction, HT29-APC cells gradually stopped proliferating. By 24 hours, most cells rounded up. By 48 hours, a substantial portion of them detached and were found floating in the culture medium and appeared apoptotic.

In parallel to a progressive decrease in survivin, we analyzed the degree of ABK activity after induction of wild type $A P C$ expression. Using reverse transcription-PCR, we assessed ABK expression and activity in HT29-APC cells. While both reverse transcription-PCR and western blots showed no change in ABK levels after induction of wild-type APC expression (data not shown), we did observe a decrease in ABK activity, specifically in the ability of immunoprecipitated-ABK to phosphorylate exogenous histone-H3 (Figure 6B). Also, endogenous phospho-histone-H3 levels decreased after induction of wild-type APC (Figure 6C). Phospho-CENP-A levels also decreased (Figure 6D).

\section{Transfection of Dominant Negative TCF-4}

In experiments designed to determine the effect of transfecting dominant negative (dn)TCF-4, using a construct shown to down-regulate survivin, ${ }^{1}$ on ABK in HT-29 cells, we observed that $A B K$ expression did not change over 24 hours (data not shown). In contrast, transfection of dnTCF-4 led to a decrease in ABK activity, as shown by the ability of immunoprecipitated-ABK to phosphorylate exogenous histone-H3 (Figure 7A). Also, endogenous phospho-H3 levels decreased after transfection of dnTCF-4. Phospho-CENP-A levels also decreased (Figure 7, B and C).

\section{Transfection of siRNAs against TCF-4 and Survivin}

The effect of TCF-4 inhibition on HT29 cells was also examined by transfecting siRNA against TCF-4. Immunoblot analysis of RNA interference (RNAi) showed that siRNA against TCF-4 substantially repressed expression of TCF-4 protein in HT29 cells (data not shown). As in strategies (1) and (2) above, expression of survivin and phospho-H3 protein decreased in parallel (not shown). When we used siRNA against survivin, expression of survivin mRNA decreased (Figure $8 \mathrm{~A}$ ) and expression of survivin and phospho-H3 proteins also decreased (Figure 8, B and C). 


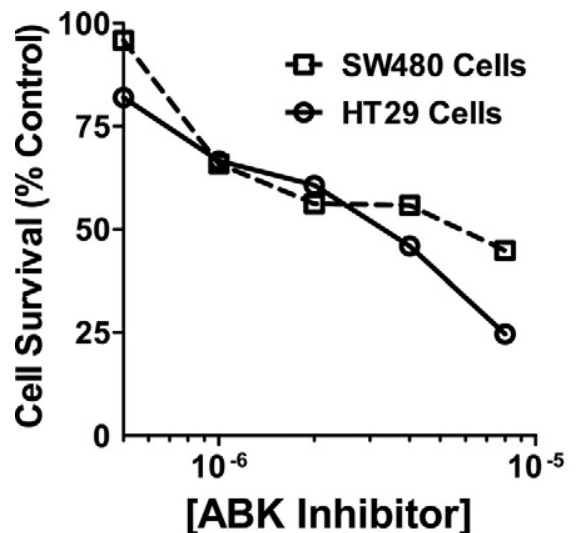

\section{Discussion}

In the above study, several independent lines of evidence for normal colonic tissues are consistent with a mechanism in which APC (a tumor-suppressor protein) controls mitosis and cell proliferation by preventing survivin-induced $A B K$ activation. In this view, if wild-type

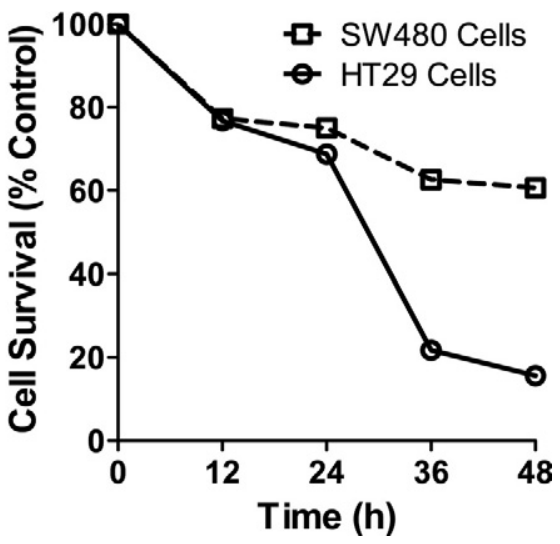

Figure 5. ABK activity is necessary for cell proliferation in cells with mutant APC. ZM447439, a known inhibitor of Aurora B kinase, ${ }^{32,33}$ inhibited the growth of colon cancer cells in vitro. SW480 and HT29 cells with a mutation in APC and overexpression of survivin were treated with ZM447439 at the indicated doses and times. The $\mathrm{IC}_{50}$ value for SW480 cells was approximately $6.125 \mu \mathrm{mol} / \mathrm{L}$; for HT29 cells, it was 3.4 $\mu \mathrm{mol} / \mathrm{L}$. As indicated in the time course experiments, treatment of these colon cancer cell lines with ZM447439 resulted in a decrease of 35\% and $85 \%$ in cell proliferation in SW 480 and HT29 cells, respectively, after 48 hours of treatment at the $\mathrm{IC}_{50}$.
A

Survivin Expression After APC Induction

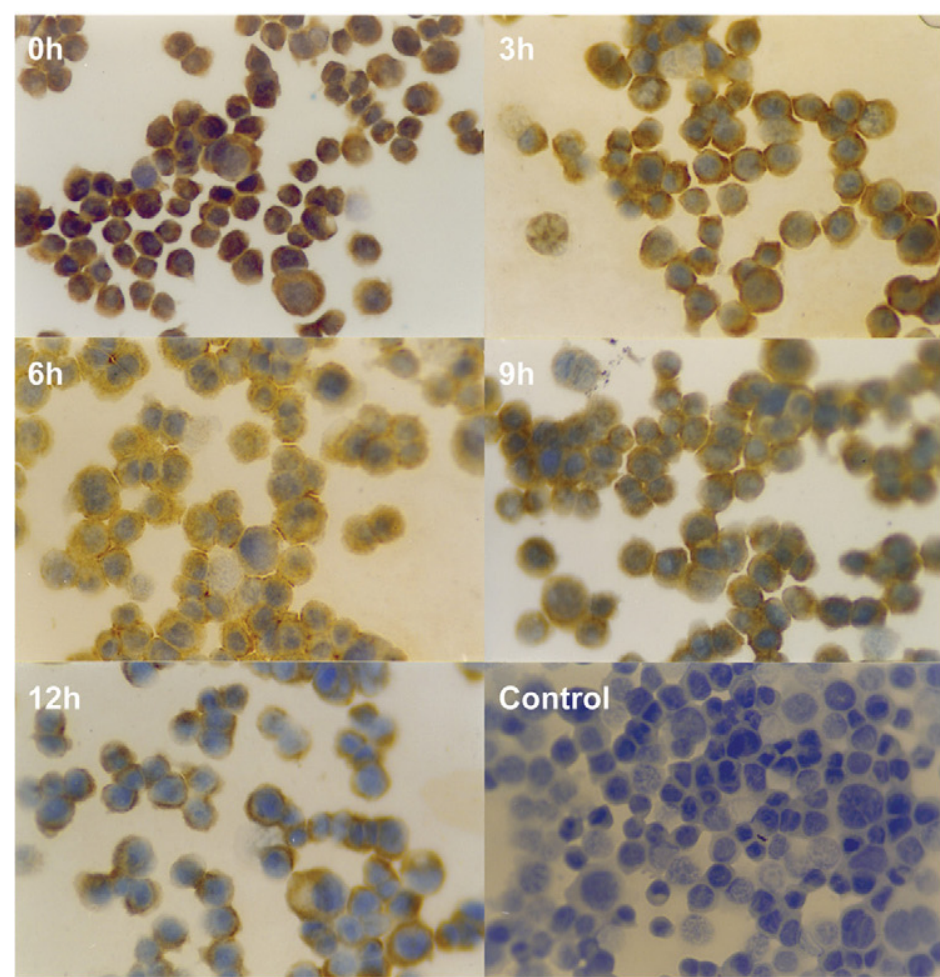

B

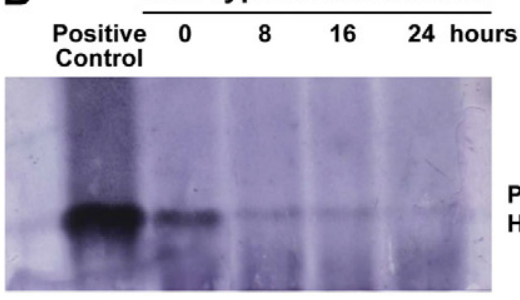

Phospho-Histone H3 (exogenous)
HT29 APC Cell (APC Inducible)

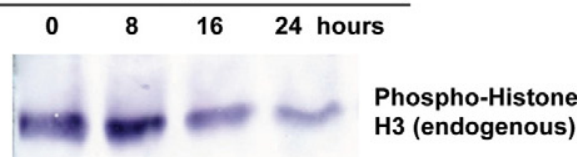

$\beta$-actin

D
HT29 APC Cell (APC Inducible)

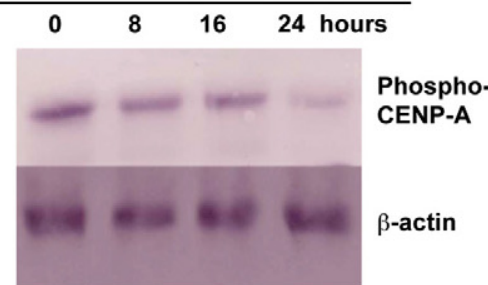

Figure 6. Inhibiting survivin signaling by induction of wild-type APC. A: Immunocytochemical staining of HT29-APC cells for survivin expression showed that induction of wild-type APC decreases survivin protein expression. The figure shows the time course for changes in survivin immunoreactivity after induction of wild-type APC by $\mathrm{ZnCl}_{2}$ treatment in $\mathrm{HT} 29$-APC cells $(0,3,6,9$, and12 hours). Negative controls involved the omission of the primary antibody in the staining of HT-29-APC cells (no staining found, data not shown) and omission of zinc treatment to induce wild-type APC expression (lower right panel shows control culture after 12 hours). Densitometry showed that survivin levels decreased by $64 \%$ at 3 hours, $84 \%$ at 6 hours, $89 \%$ at 9 hours, and $93 \%$ at 12 hours after the induction of wild-type APC expression in HT29 cells. Original magnification $\times 200$. B: In parallel, induction of wild-type APC expression led to decreased ABK activity in the HT29-APC colon cancer cell line. After immunoprecipitating ABK in HT29 cells, ABK kinase activity was measured by the ability of immunoprecipitated ABK to phosphorylate exogenous phospho-histone-H3. A similar result was found for (C) endogenous phospho-histone-H3 and (D) phospho-CENP-A, as both were decreased, indicating lower endogenous ABK enzymatic activity. ABK mRNA and protein, and unphosphorylated histone-H3 and CENP-A protein levels did not change (data not shown). Densitometry showed that ABK activity was decreased by $82 \%$ at 8 hours, $87 \%$ at 16 hours, and $92 \%$ at 24 hours (B), endogenous phospho-histone-H3 decreased by $22 \%$ at 8 hours, $55 \%$ at 16 hours, and $63 \%$ at 24 hours (C), and endogenous phospho-CENP-A decreased by $34 \%$ at 8 hours, $55 \%$ at 16 hours, and $80 \%$ at 24 hours (D) after induction of wild-type APC expression in HT29 cells. 
A
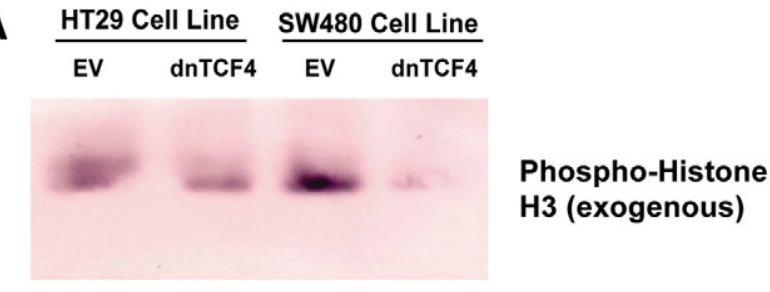

\section{B HT29 Cell Line sW480 Cell Line}

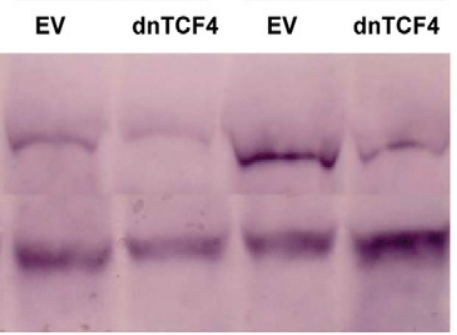

Phospho-Histone H3 (endogenous)

\section{$\beta$-actin}
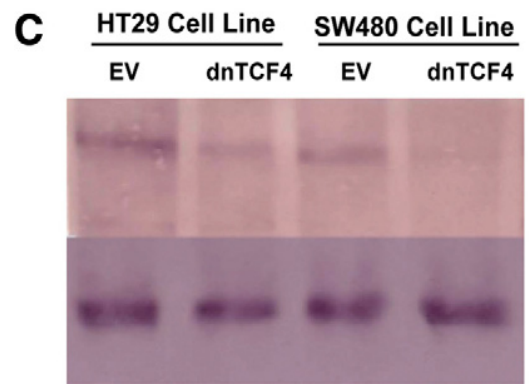

Phospho-CENP-A

Figure 7. Inhibiting survivin signaling by transfection of dominant-negative TCF-4. When a dnTCF-4 construct or empty vector (EV) was stably transfected into HT29 or SW480 cells, ABK activity decreased as indicated by decreased phosphorylation of exogenous phospho-histone-H3 using immunoprecipitated ABK (A) and lower endogenous phospho-histone-H3 (B). C: Endogenous phospho-CENP-A levels also decreased. Densitometry showed that ABK activity decreased by 52\% in HT29 cells and $86 \%$ in SW480 cells, endogenous phospho-H3 levels decreased by 53\% n HT29 cells and 75\% in SW480 cells, and endogenous phospho-CENP-A levels decreased by $55 \% \mathrm{n}$ HT29 cells and 64\% in SW480 cells.

high. And that is what we found. Immunostaining, western blotting and co-immunoprecipitation experiments all indicated similar intracryptal gradients-highest at the bottom and diminishing toward the crypt top-for the ABK complex and three of its components, survivin, INCENP and $A B K$, as well as for ABK activity and its phosphorylated substrates $\mathrm{H} 3$ and CENP-A. This pattern was the inverse of the observed APC gradient (Figure 3, B and C, Reference 11), which was lowest at the bottom and highest at the crypt top. If our mechanism is correct, ABK signaling in the crypt should colocalize with populations of mitotic and proliferating cells, and this, too, was found to be the case-ABK+, phospho-H3+, and $\mathrm{Ki}-67+$ cell populations were mostly found in the lower crypt.

We also found that in cells with an APC mutation, survivin expression is up-regulated and induction of wild type APC expression in these cells down-regulates survivin expression. These findings indicate that survivin is a TCF-4 target gene. In contrast, our results on the effects of TCF-4 inactivation, using three independent approaches, showed that inhibiting TCF-4 signaling in cells does not reduce ABK expression, it reduces ABK activity. Thus, our results do not indicate that $A B K$ is a TCF4 target
A

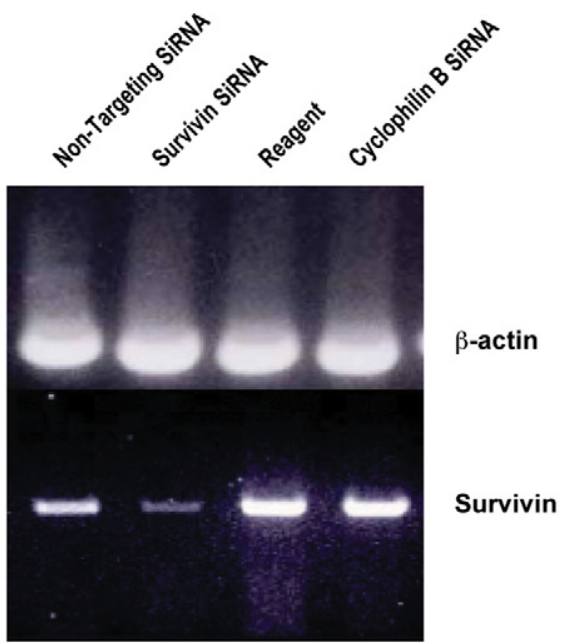

B

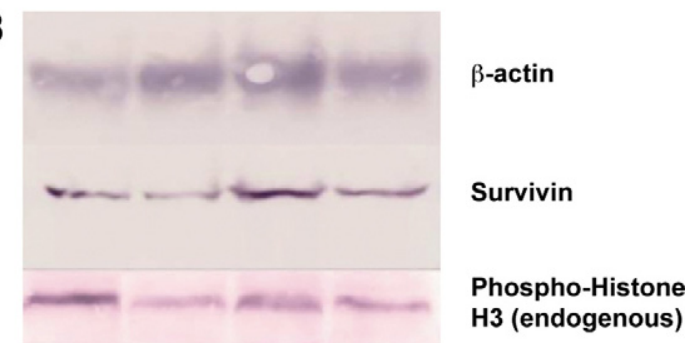

Figure 8. Inhibiting survivin signaling via inactivation of survivin by siRNA A: When siRNA against survivin was transfected into HT29 cells, survivin mRNA expression decreased as determined by RT-PCR (Lane $1=$ nontargeting siRNA; Lane 2 = survivin siRNA; Lane 3 = transfection reagent only; Lane $4=$ cyclophilin B siRNA). B and C: In the same cells, a similar decrease was observed for survivin protein and endogenous phospho-histone- $\mathrm{H} 3$ protein levels as determined by Western blot analysis. Densitometry showed that for survivin, the RNA level decreased by $68 \%$, survivin protein by $61 \%$, and endogenous phospho-histone-H3 levels by $66 \%$ after anti-survivin siRNA was transfected into HT29 cells.

gene or that $A P C$ transcriptionally controls ABK expression via TCF-4, as it does survivin expression.

Our proposed mechanism requires that survivin and ABK have a role in controlling mitosis, and that appears to be the case. ABK activation requires association of survivin, ABK, and INCENP.4,5,27,28 Once associated, ABK phosphorylates specific substrates such as histone-H3 and CENP-A, which are necessary for mitosis. $^{29,30}$ Evidence also indicates that INCENP and ABK act together with survivin at the beginning of and throughout mitosis in a chromosomal passenger complex. ${ }^{31}$ For example, Carvalho et $\mathrm{al}^{23}$ showed that after transfection of cells with survivin siRNA to deplete survivin levels, cells without detectable survivin also lacked centromeric ABK and INCENP. This revealed a linear correlation between levels of survivin and ABK and INCENP at centromeres. Thus, it appears that survivin is required for $A B K$ and INCENP to localize to centromeres. Down-regulation of survivin by transfection of antisense oligonucleotides also leads to a cytokinesis defect. ${ }^{4}$ Moreover, both immunostaining for endogenous survivin and ectopic expression of green fluorescent protein-tagged survivin showed that survivin is colocalized with ABK and INCENP to the cleavage furrow during late mitosis. 8,27 Thus, the association of survivin, ABK, and INCENP is necessary for the proper 
segregation of replicated chromosomes in mitosis, which must be precisely coordinated in space and time during cytokinesis. $5,23,27,28$

Our findings for normal crypts also suggest that $A P C$, via survivin signaling, may be involved in regulation of (i) SC dynamics and crypt cell renewal, (ii) size of proliferative cell populations, and (iii) crypt cell maturation. For example, we found in the present study that cells that stained positively for the SC marker ALDH1 are survivinnegative and, in normal crypts, reside below the survivin + cell population. In comparison, proliferating (Ki-67 positive) cells are survivin-positive, and ABK-active as indicated by the presence of phospho-H3. This indicates that activation of ABK in non-SC offspring is due to survivin expression. These findings provide an explanation for why the proliferating, $\mathrm{Ki}-67+$, population is restricted to the lower region of the normal crypt. Namely, this distribution may be due to $A P C$-induced cell maturation and differentiation as cells migrate up the crypt. In such a case, the loss of proliferative ability may be due to increasing concentrations of APC that (via inactivation of TCF-4) down-regulate survivin and lower ABK activity. Indeed, we found that survivin levels and ABK activity decreased toward the crypt top. This loss of survivin expression and $A B K$ activity will cause cells to lose their ability to proliferate. In this way, $A P C$-induced cell maturation could govern the size of the proliferative cell population and ultimately contribute to terminal differentiation of crypt cells in the upper crypt.

Our proposed mechanism not only suggests how APC controls mitosis/proliferation in normal cells, but also, it provides a possible explanation for how an APC mutation helps initiate and promote colon tumorigenesis. Broadly, the explanation is that $A P C$ mutation leads to disinhibition of survivin expression and activation of $A B K$, which results in increased mitosis and proliferation, two cardinal signs of colon tumorigenesis. Our data showing that ZM447439, a known ABK inhibitor, ${ }^{32,33}$ decreases the proliferation of colon cancer cells that are known to have mutant $A P C$ (and overexpression of survivin), provides evidence that $A B K$ activity is required for cell proliferation. Moreover, we found that induction of wild type APC in colon carcinoma cells that have mutant $A P C$ and overexpression of survivin, not only down-regulates survivin expression and ABK activity, but also, it attenuates cell proliferation (Figure 6; reference 34). These results suggest that survivin expression and its ability to promote mitosis via activation of $A B K$ may play a role not only in cells from advanced colon tumors with biallelic mutant APC, but also in cells that develop monoallelic APC mutations at the very earliest stages of neoplasia.

Supporting this view that ABK activity is required for tumor cell proliferation is the report that increased histone-H3 Ser-10 phosphorylation, an indicator of ABK activation, was observed in various colorectal cancer cells that express ABK. ${ }^{35}$ Moreover, most CRCs harbor APC mutations and overexpress survivin. ${ }^{12}$ Indeed, our data on immunostaining for survivin signaling components in neoplastic tissues (normal appearing FAP crypts, adenomatous crypts) provide a more detailed explanation.
For FAP crypts, which have a germline APC mutation, we found that the populations of cells that expressed survivin, $\mathrm{ABK}$ and phospho-H3 were extended upward into the middle and upper crypt regions. In adenomatous crypts, which have bi-allelic APC mutations, populations of cells positive for all ABK-related proteins were extended even further up the crypt. These finding are parallel to changes we saw for the proliferating $\left(\mathrm{Ki}^{-} 67^{+}\right)$cell population, which also extended upwards in FAP crypts and even further in adenomatous crypts. Similar observations were previously reported for bromodeoxyuridine and $\left[{ }^{3} \mathrm{H}\right]$ thymidine labeling of colonic crypts, ${ }^{17-20}$ whereby shifts in the labeling indices (for S phase cells) were also discovered in FAP and adenomatous crypts. This upward-shifting of transitions between crypt cell phenotypes-from stem (survivin-negative/ABK inactive) to proliferating (survivin-positive/ABK active) to terminally differentiated (survivin-negative/ABK inactive) to apoptotic cells-indicates that survivin signaling becomes dysregulated in a way that delays maturation of cells migrating up the crypt.

The above study on survivin signaling proteins in human colonic crypts indicates that the organization of proliferative cell populations undergoes dramatic changes during CRC development. These findings support our hypotheses that (i) in normal human colonic crypts wild-type $A P C$ down-regulates ABK activity and (ii) in neoplastic human crypts, where APC is mutant, survivin is overexpressed and $A B K$ is up-regulated and associated with increased proliferation of SCs and proliferating cells.

In normal colonic crypts, the population of cells staining for the ALDH1 stem cell marker and the subpopulation of cells staining for survivin and other proliferative markers are both restricted to the lower crypt. In neoplastic crypts, both the subpopulation of stem cells $(\mathrm{ALDH} 1+)$ and the subpopulation of proliferating cells (Ki-67+, survivin+, ABK+, phospho-H3+) are expanded and distributed further up the crypt. This provides biological evidence (shown in Figures 1 and 2) in support of our prediction from our mathematical modeling studies ${ }^{14-16}$ that the SC population (which is at the crypt bottom) is expanded, and this SC overpopulation causes expansion and upward shifting of the proliferating cell population toward the top of neoplastic crypts.

In this view, dysregulation of mechanisms controlling survivin signaling (eg, by APC mutations) delays maturation, allows expansion of the SC population and then the proliferating cell population in the crypt, and contributes to colon tumorigenesis. This effect may be synergistic with survivin's ability to prevent apoptosis; both effects would promote tumor growth. Ultimately, both these mechanisms contribute to the exponential increase in proliferative cell populations, including mitotic cells, which are hallmarks of CRC pathology. ${ }^{15}$ Key questions remaining for future studies are: (i) how are survivin expression and ABK activation suppressed in SCs at the crypt bottom, which lack detectable levels of APC; (ii) does dysregulation of this mechanism in stem cells contribute to their overpopulation in neoplasia and colonic neoplasms? 


\section{Acknowledgments}

We thank the Thomas Jefferson University Pathology Core Facility personnel for their assistance and Michael Schnee for editorial assistance in manuscript writing. We also thank Tim Flanagan for assistance with figure preparation. We thank Dr. Bert Vogelstein and Dr. Kenneth Kinzler for providing the recombinant HT29 cell lines; Dr. William C. Earnshaw for providing the anti-INCENP antibody; and Kim Henderson, Zenobia Cofer, and Shirish Damle for technical assistance.

\section{References}

1. Zhang T, Otevrel T, Gao Z, Ehrlich SM, Fields JZ, Boman BM: Evidence that APC regulates survivin expression: a possible mechanism contributing to the stem cell origin of colon cancer. Cancer Res 2001, 61:8664-8667

2. Boman B, Kopelovich L, Siracusa LD, Zhang T, Henderson K, Cofer Z, Buchberg AM, Fields JZ, Otevrel T: A GFP-Tcf4 reporter mouse model for monitoring effects of Apc mutations during intestinal tumorigenesis. Mol Carcinog 2009, 48:821-831

3. Boman B, Walters R, Fields JZ, Kovatich AJ, Zhang T, Isenberg GA, Goldstein SD, Palazzo JP: Colonic crypt changes during adenoma development in familial adenomatous polyposis: immunohistochemical evidence for expansion of the crypt base cell population. Am J Pathol 2004, 165:1489-1498

4. Chen J, Jin S, Tahir SK, Zhang H, Liu X, Sarthy AV, McGonigal TP, Liu Z, Rosenberg SH, Ng SC: Survivin enhances Aurora-B kinase activity and localizes Aurora-B in human cells. J Biol Chem 2003, 278:486-490

5. Bolton MA, Lan W, Powers SE, McCleland ML, Kuang J, Stukenberg PT: Aurora B kinase exists in a complex with survivin and INCENP and its kinase activity is stimulated by survivin binding and phosphorylation. Mol Biol Cell 2002, 13:3064-3077

6. Katayama H, Ota T, Jisaki F, Ueda Y, Tanaka T, Odashima S, Suzuki F, Terada Y, Tatsuka M: Mitotic kinase expression and colorectal cancer progression. J Natl Cancer Inst 1999, 91:1160-1162

7. Tatsuka M, Katayama H, Ota T, Tanaka T, Odashima S, Suzuki F, Terada $Y$ : Multinuclearity and increased ploidy caused by overexpression of the aurora- and Ipl1-like midbody-associated protein mitotic kinase in human cancer cells. Cancer Res 1998, 58:4811-4816

8. Adams RR, Eckley DM, Vagnarelli P, Wheatley SP, Gerloff DL, Mackay AM, Svingen PA, Kaufmann SH, Earnshaw WC: Human INCENP colocalizes with the Aurora-B/AIRK2 kinase on chromosomes and is overexpressed in tumour cells. Chromosoma 2001, 110:65-74

9. Ota T, Suto S, Katayama H, Han ZB, Suzuki F, Maeda M, Tanino M, Terada Y, Tatsuka M: Increased mitotic phosphorylation of histone H3 attributable to AIM-1/Aurora-B overexpression contributes to chromosome number instability. Cancer Res 2002, 62:5168-5177

10. Boman BM, Zhang T, Fields JZ: Evidence that APC regulates survivin expression: a possible mechanism contributing to the stem cell origin of colon cancer. (letter to the editor) Cancer Res 2004, 64:776-779

11. Smith KJ, Johnson KA, Bryan TM, Hill DE, Markowitz S, Willson JK, Paraskeva C, Petersen GM, Hamilton SR, Vogelstein B: The APC gene product in normal and tumor cells. Proc Natl Acad Sci USA 1993, 90:2846-2850

12. Kim PJ, Plescia J, Clevers H, Fearon ER, Altieri DC: Survivin and molecular pathogenesis of colorectal cancer. Lancet 2003, 362:205-209

13. Ma H, Nguyen $\mathrm{C}$, Lee KS, Kahn M: Differential roles for the coactivators CBP and p300 on TCF/beta-catenin-mediated survivin gene expression. Oncogene 2005, 24:3619-3631

14. Boman BM, Fields JZ, Bonham-Carter O, Runquist OA: Computer modeling implicates stem cell overproduction in colon cancer initiation. Cancer Res 2001, 61:8408-8411

15. Boman BM, Wicha MS, Fields JZ, Runquist OA: Symmetric division of cancer stem cells-a key mechanism in tumor growth that should be targeted in future therapeutic approaches. Clin Pharmacol Ther 2007, 81:893-898

16. Boman BM, Fields JZ, Cavanaugh KL, Gujetter A, Runquist OA: How dysregulated colonic crypt dynamics cause stem cell overpopulation and initiate colon cancer. Cancer Res 2008, 6:3304-3313

17. Deschner $\mathrm{E}$, Lewis CM, Lipkin $\mathrm{M}$ : In vitro study of human rectal epithelial cells. I atypical zone of $\mathrm{H} 3$ thymidine incorporation in mucosa of multiple polyposis. J Clin Invest 1963, 42:1922-1928

18. Lightdale C, Lipkin M, Deschner E: In vivo measurements in familial polyposis: kinetics and location of proliferating cells in colonic adenomas. Cancer Res 1982, 42:4280-4283

19. Potten CS: Stem cells in gastrointestinal epithelium: numbers, characteristics and death. Philos Trans R Soc Lond B Biol Sci 1998, 353:821-830

20. Potten CS, Kellett M, Rew DA, Roberts SA: Proliferation in human gastrointestinal epithelium using bromodeoxyuridine in vivo: data for different sites, proximity to a tumour, and polyposis coli. Gut 1992 33:524-529

21. Wildrick DM, Lointier P, Nichols DH, Roll R, Quintanilla B, Boman BM: Isolation of normal human colonic mucosa: comparison of methods. In Vitro Cell Dev Biol Anim 1997, 33:18-27

22. Huang EH, Hynes MJ, Zhang T, Ginestier C, Dontu G, Appelman H Fields JZ, Wicha M, Boman BM: ALDH1 is a marker for normal and malignant human colonic stem cells and tracks stem cell overpopulation during colon tumorigenesis. Cancer Res 2009, 69:3382-3389

23. Carvalho A, Carmena M, Sambade C, Earnshaw WC, Wheatley SP: Survivin is required for stable checkpoint activation in taxol-treated HeLa cells. J Cell Sci 2003, 116:2987-2998

24. Zeitlin SG, Shelby RD, Sullivan KF: CENP-A is phosphorylated by Aurora B kinase and plays an unexpected role in completion of cytokinesis. J Cell Biol 2001, 155:1147-1157

25. Wei Y, Yu L, Bowen J, Gorovsky MA, Allis CD: Phosphorylation of histone $\mathrm{H} 3$ is required for proper chromosome condensation and segregation. Cell 1999, 97:99-109

26. Adams RR, Maiato H, Earnshaw WC, Carmena M: Essential roles of Drosophila inner centromere protein (INCENP) and aurora B in histone $\mathrm{H} 3$ phosphorylation, metaphase chromosome alignment, kinetochore disjunction, and chromosome segregation. J Cell Biol 2001, 153:865-880

27. Wheatley SP, Carvalho A, Vagnarelli P, Earnshaw WC: INCENP is required for proper targeting of Survivin to the centromeres and the anaphase spindle during mitosis. Curr Biol 2001, 11:886-890

28. Romano A, Guse A, Krascenicova I, Schnabel H, Schnabel R, Glotzer M: CSC-1: a subunit of the Aurora B kinase complex that binds to the survivin-like protein BIR-1 and the incenp-like protein ICP-1. J Cell Biol 2003, 161:229-236

29. Juan G, Traganos F, James WM, Ray JM, Roberge M, Sauve DM, Anderson H, Darzynkiewicz Z: Histone H3 phosphorylation and expression of cyclins A and B1 measured in individual cells during their progression through $\mathrm{G} 2$ and mitosis. Cytometry 1998, 32:71-77

30. Crosio C, Heitz E, Allis CD, Borrelli E, Sassone-Corsi P: Chromatin remodeling and neuronal response: multiple signaling pathways induce specific histone $\mathrm{H} 3$ modifications and early gene expression in hippocampal neurons. J Cell Sci 2003, 116:4905-4914

31. Leverson JD, Huang HK, Forsburg SL, Hunter T: The Schizosaccharomyces pombe aurora-related kinase Ark1 interacts with the inner centromere protein Pic1 and mediates chromosome segregation and cytokinesis. Mol Biol Cell 2002, 13:1132-1143

32. Girdler F, Gascoigne KE, Eyers PA, Hartmuth S, Crafter C, Foote KM, Keen NJ, Taylor SS: Validating Aurora B as an anti-cancer drug target. J Cell Sci 2006, 119:3664-3675

33. Ditchfield C, Johnson VL, Tighe A, Ellston R, Haworth C, Johnson T, Mortlock A, Keen N, Taylor SS: Aurora B couples chromosome alignment with anaphase by targeting BubR1, Mad2, and Cenp-E to kinetochores. J Cell Biol, 2003, 161:267-280

34. Morin PJ, Vogelstein B, Kinzler KW: Apoptosis and APC in colorectal tumorigenesis. Proc Natl Acad Sci USA 1996, 93:7950-7954

35. Takahashi T, Futamura M, Yoshimi N, Sano J, Katada M, Takagi Y, Kimura M, Yoshioka T, Okano Y, Saji S: Centrosomal kinases. HSAIRK1 and HsAIRK3, are overexpressed in primary colorectal cancers. Jpn J Cancer Res 2000, 91:1007-1014 